\title{
Diversity Analysis of Actinomycetes Assemblages Isolated from Soils in Cool-temperate and Subtropical Areas of Japan
}

\author{
Masayuki Hayakawa $^{1 *}$, Hideki Yamamura ${ }^{1}$, Yuuya Sakuraki ${ }^{1}$, Yuumi Ishida ${ }^{2}$, \\ Moriyuki Hamada ${ }^{2}$, Misa Otoguro ${ }^{2}$ and Tomohiko Tamura ${ }^{2}$ \\ ${ }^{1}$ Division of Applied Biological Sciences, Interdisciplinary Graduate School of Medicine and Engineering, \\ University of Yamanashi, Takeda-4, Kofu 400, Japan \\ ${ }^{2}$ NITE Biological Resource Center (NBRC), National Institute of Technology and Evaluation, \\ 2-5-8 Kazusakamatari, Kisarazu, Chiba 292-0818, Japan
}

(Received Oct. 13, 2009 / Accepted Mar. 17, 2010 / Published May 17, 2010)

\begin{abstract}
The taxonomic diversity of actinomycetes from soils in a cool-temperate area (74 samples from Rishiri Island) and a subtropical area (94 samples from Iriomote Island) of Japan was compared. Soil samples were treated with five different pre-treatment methods and subsequently cultured on humic acid-vitamin agar, resulting in the selective isolation of 1,234 actinomycete strains. These isolates were classified using $16 \mathrm{~S}$ rDNA sequence analysis. 16S rDNA sequence similarity values of $\geq 97 \%$ and $\geq 98.7 \%$ were tentatively used to identify isolates at the genus and species ranks, respectively. The actinomycete assemblages isolated comprised diverse species. Specifically, 668 of the Rishiri isolates fell into 17 families, 40 genera, and 178 species, and 566 of the Iriomote isolates belonged to 21 families, 59 genera, and 194 species. Diversity analyses based on the Shannon index revealed that there was no significant difference between the species diversity of the Rishiri isolates and that of the Iriomote isolates. However, there was little taxonomic overlap between the Rishiri isolates and the Iriomote isolates, as only 66 of the species occurred in both groups. Several species that were reportedly isolated from other tropical areas were found among the Iriomote isolates but not among the Rishiri isolates. This result suggests that climate may influence the species types of soil-inhabiting actinomycetes.
\end{abstract}

\section{INTRODUCTION}

Actinomycetes usually form a significant component of the microbial population in soil, and play a significant role in the digestion and recycling of more recalcitrant, naturally occurring organic compounds (Goodfellow \& Williams, 1983). The abundance and types of these microorganisms in soil habitats vary greatly with ecological factors such as vegetation, soil $\mathrm{pH}$, humus content, and water content (Williams et al., 1984). Climate is also thought to be an important ecological factor influencing soil actinomycete flora, although there have been few comparisons of the actinomycete populations among areas with different climates. Xu et al. (1996) studied the distribution of actinomycetes in soils of Yunnan, China, and reported that their generic diversity was more complex in subtropical and tropical areas than in cool-temperate mountain and snow-mountain areas. More recently, Muramatsu et al. (2003) studied the taxonomic diversity of actinomycetes from forest-soils in tropical (Selangor, Malaysia) and temperate (Tokyo, Japan) areas. Using a simple identification method based on 16S rDNA partial sequence similarity, they demonstrated that actinomycetes belonging to diverse genera and species were present in both areas. Moreover, they found little taxonomic overlap between Malaysian and Japanese isolates.
The aim of this study was to investigate and compare taxonomic diversity at the genus and species level of actinomycetes from soils in cool-temperate and subtropical areas of Japan. Five different isolation methods were used to isolate actinomycetes from soils obtained from a cooltemperate area on Rishiri Island and a subtropical area on Iriomote Island. More than 1,000 actinomycetes were isolated. These were classified using a simple method based on analysis of nearly complete $16 \mathrm{~S}$ rDNA sequences.

\section{MATERIALS AND METHODS}

\section{Soil samples}

A total of 74 samples ( 25 field soils, 31 forest soils, and other 18 soils from riverside, seashore, etc.) were collected from Rishiri, a cool-temperate island $\left(182 \mathrm{~km}^{2}\right.$ in total area) located north-west of Hokkaido at $45.1^{\circ} \mathrm{N}$ and $141.1^{\circ} \mathrm{E}$ (Fig. 1). Annual mean temperature and annual rainfall in 2007 were $7.4^{\circ} \mathrm{C}$ and $859 \mathrm{~mm}$, respectively (Japan Meteorological Agency: http://www.jma.go.jp/jma/index.html). Similarly, 95 samples (41 field soils, 12 forest soils, and other 42 soils from riverside, seashore, etc.) were collected at Iriomote, a subtropical island $\left(289 \mathrm{~km}^{2}\right.$ in total area) located east of Taiwan at $24.2^{\circ} \mathrm{N}$ and $123.5^{\circ} \mathrm{E}$ (Fig. 1). Annual mean temperature and annual rainfall in 2007 were $24.2^{\circ} \mathrm{C}$ and $2,485 \mathrm{~mm}$, respectively (JMA). Samples were

${ }^{*}$ Corresponding author. Phone: +81-55-220-8542. Fax: +81-55-220-8543. E-mail: hayakawa@ab11.yamanashi.ac.jp 


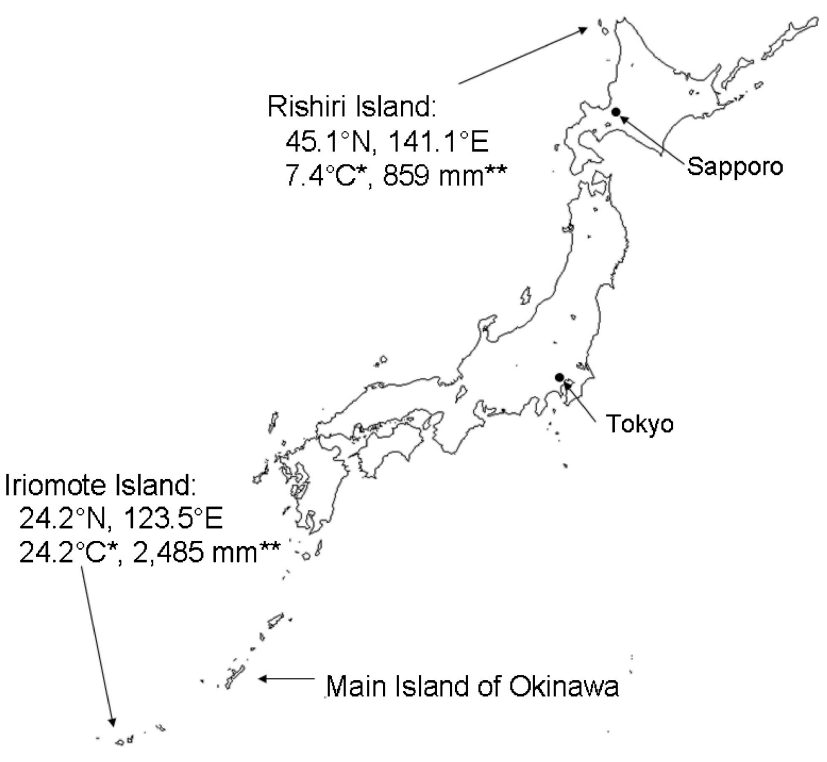

Fig. 1. Sampling areas (Rishiri Island and Iriomote Island) in Japan.

* Annual mean temperature in 2007. ** Annual rainfall in 2007.

collected from July to September of 2007 and 2008. Each sample was passed through a 2-mm mesh sieve, air-dried at room temperature for 7 days, and subsequently used for isolation of actinomycetes (Hayakawa et al., 1988).

\section{Isolation procedures for actinomycetes}

Actinomycetes were isolated from soil samples using five types of pre-treatment methods; the SDS method (Hayakawa \& Nonomura, 1988), the dry-heating method (Nonomura \& Ohara, 1969a; Hayakawa et al., 1991a), the rehydration and centrifugation method (Hayakawa et al., 2000), the chemotactic method (Hayakawa et al., 1991b), and the air-drying method (Hayakawa et al., 1989). Humic acid-vitamin (HV) agar (Hayakawa \& Nonomura, 1987) supplemented with nalidixic acid $(20 \mu \mathrm{g} / \mathrm{ml}$; Sigma Chemical Co., St Louis, MO, USA) and cycloheximide $(50 \mu \mathrm{g} / \mathrm{ml}$; Wako Pure Chemical Ind., Osaka, Japan) was used as the isolation medium. Where appropriate, $\mathrm{NaCl}(5$ or $10 \%)$ or sorbitol (10\%) was added to HV agar. All plates were incubated at $30^{\circ} \mathrm{C}$ for $2-3$ weeks. Representative actinomycete colonies growing on the isolation plates were purified by streaking onto Bennett's agar (Jones, 1949). A total of 1,234 actinomycete strains were thus obtained in pure culture.

\section{Taxonomic analyses}

Strains. All actinomycete isolates (1,234 strains) were stored on oatmeal-YGG agar (Hayakawa et al., 1982) or yeast-starch agar (Nonomura \& Ohara, 1969b) slants and used for taxonomic characterization.

Morphological characterization. Actinomycete isolates were grown on HA agar (Nonomura et al., 1979), or on
oatmeal-YGG agar. Morphology was observed by using a light microscope or a scanning electron microscope (JSM6500F, Jeol Ltd., Tokyo) under previously described conditions (Hayakawa et al., 1996).

Phylogenetic analysis. Genomic DNA was extracted using PrepMan ${ }^{\circledR}$ ultra reagent (Applied Biosystems, CA, USA) according to the manufacturer's protocol. A nearly complete $16 \mathrm{~S}$ rDNA sequence $(1.5 \mathrm{~Kb}$; positions $22-1528$, according to the Escherichia coli numbering system; Brosius et al., 1978) was amplified from each isolate by PCR following the procedure described by Tamura \& Hatano (2001) and directly sequenced using an ABI Prism BigDye Terminator cycle sequencing kit (Applied Biosystems) and an ABI Model 3730 automatic DNA sequencer.

The 16S rDNA sequences determined over $1,200 \mathrm{bp}$ were compared with published sequences in the TYP16 database (Chun et al., 2007) using a web-based identification tool, EzTaxon, which performs similarity-based searches (http://www.eztaxon.org). BLAST searches of the EMBL/GenBank/DDBJ database were also performed to confirm the results. Phylogenetic identity of each actinomycete isolate was then verified by constructing and evaluating phylogenetic trees. Specifically, the $16 \mathrm{~S}$ rDNA sequences obtained from the isolates were aligned with reference sequences of a corresponding genus available from EMBL/GenBank/DDBJ using the CLUSTAL_X program (Thompson et al., 1997). A phylogenetic tree was constructed using neighbor-joining (NJ) tree algorithms (Saitou \& Nei, 1987). The resultant NJ tree topology was evaluated by bootstrap analysis based on 1,000 replicates (Felsenstein, 1985).

Identification to genus and species level. Based on the studies of Drancourt et al. (2000) and Stackebrandt \& Ebers (2006), we defined 16S rDNA sequence similarity values of $\geq 97 \%$ and $\geq 98.7 \%$ as the criteria for identification at the genus and species levels, respectively. More specifically, identification to the genus rank was defined as 16S rDNA sequence similarity of $\geq 97 \%$ to that of the prototype strain sequence in the TYP16S database. Similarly, if an isolate showed $\geq 98.7 \% 16 \mathrm{~S}$ rDNA sequence similarity to the most homologous prototype species, the isolate was classified as the same species as that prototype species.

For those isolates that were not sufficiently similar to any reference species to be classified using the $16 \mathrm{~S}$ rDNA-based method, taxonomic identity was inferred from the phylogenetic tree analysis. Morphological characteristics were also used to validate identity at the genus level.

\section{Diversity analysis}

The diversity of actinomycete species was examined by the Shannon diversity index (Shannon \& Weaver, 1949), a general diversity index that considers both richness and evenness. The equation for the Shannon index is as follows: $H^{\prime}=-\Sigma P_{i} \ln P_{i}$, where $P_{i}$ is the number of individuals in $i$ th species as a fraction of the total number of individuals in the sample. The Shannon diversity index was evaluated 
Table 1. Isolation of actinomycetes from soils ${ }^{\text {a) }}$ on Rishiri Island and Iriomote Island.

\begin{tabular}{|c|c|c|c|}
\hline \multirow{2}{*}{ Pre-treatment } & \multirow{2}{*}{ Culture medium ${ }^{\text {b) }}$} & \multicolumn{2}{|c|}{ No. of isolates } \\
\hline & & Rishiri Island & Iriomote Island \\
\hline SDS and Yeast extract ${ }^{\mathrm{c})}$ & HV agar & 280 & 310 \\
\hline Dry-heating ${ }^{\mathrm{d})}$ & HV agar & 80 & 48 \\
\hline Rehydration and centrifugation ${ }^{\mathrm{e})}$ & HV agar & 206 & 137 \\
\hline (hemotaxis ${ }^{\mathrm{f}}$ & HV agar & 10 & 12 \\
\hline Air-drying & $\begin{array}{l}\mathrm{HV} \text { agar with } \mathrm{NaCl} 5 \% \text {, } \\
\mathrm{NaCl} 10 \% \text {, or Sorbitol } 10 \%\end{array}$ & 92 & 59 \\
\hline
\end{tabular}

a) Rishiri Island: 74 samples ( 25 field soils, 31 forest soils, and 18 other soils from seashore, riverside, etc.). Iriomote Island: 95 samples (41 field soils, 12 forest soils, and 42 other soils from seashore, riverside, etc.).

b) Contains cycloheximide $(50 \mu \mathrm{g} / \mathrm{ml})$ and nalidixic acid $(20 \mu \mathrm{g} / \mathrm{ml})$.

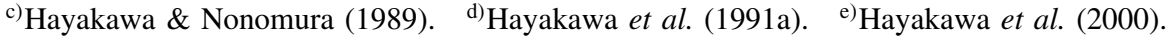

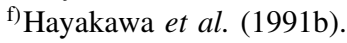

with the Wilcoxon signed-rank test for paired samples (Flanagan, et al., 2007).

\section{RESULTS}

\section{S rDNA-based identification}

We used five different pre-treatment techniques to isolate actinomycetes from soils from Rishiri, a cool-temperate island, and Iriomote, a subtropical island. This resulted in the isolation of 1,234 actinomycete strains comprising 668 Rishiri isolates and 566 Iriomote isolates (Table 1). We sequenced more than $1,200 \mathrm{bp}$ of $16 \mathrm{~S}$ rDNA from all isolates and subsequently performed taxonomic classification to the genus and species levels. The 16S rDNA sequence similarity values of $\geq 97 \%$ and $\geq 98.7 \%$ were used for simplified identification of isolates at the genus and species ranks, respectively. This 16S rDNA-based method enabled the identification of 1,215 (98.5\%) and $923(74.8 \%)$ of 1,234 actinomycete isolates to genus and species levels, respectively. For those isolates that could not be identified using this method, a phylogenetic tree analysis was used to infer their taxonomic positions at the family and genus levels.

\section{Taxonomic diversity}

Actinomycete isolates fell into a variety of distantly related families, genera, and species. The Rishiri isolates (668 strains) fell into 17 families, 40 genera, and 178 species. Similarly, the Iriomote isolates (566 strains) belonged to 21 families, 59 genera, and 194 species. The species diversity was quantified using the Shannon index, and the resulting diversity values were compared with the Wilcoxon signed-rank test (Table 2). There was no significant difference $(\mathrm{P}<0.05)$ between the Shannon diversity index of Rishiri isolates and that of Iriomote isolates.

A total of 61 genera were found among the Rishiri and Iriomote isolates (Table 3). Of these, $38(62.2 \%)$ were found on both Rishiri and Iriomote. The most frequently
Table 2. Diversity index of actinomycete species on Rishiri (a cool-temperate island) and Iriomote (a subtropical island).

\begin{tabular}{|c|c|c|c|}
\hline \multicolumn{2}{|c|}{$\begin{array}{l}\text { No. of species } \\
\text { (No. of strains) }\end{array}$} & \multicolumn{2}{|c|}{ Shannon diversity index ${ }^{a)}$} \\
\hline Rishiri & Iriomote & Rishiri & Iriomote \\
\hline $178(507)$ & $194(416)$ & $6.83^{\mathrm{b})}$ & $7.17^{\mathrm{b})}$ \\
\hline
\end{tabular}

isolated genus was Streptomyces, followed in order by Micromonospora, Actinoplanes, Nocardia, Streptosporangium, Dactylosporangium, Rhodococcus, Kitasatospora, Nonomuraea, Actinomadura, Microbispora, Pseudonocardia, Agromyces, and Catellatospora. Twenty-one genera, including Catenuloplanes, Krasilnikovia, Verrucosispora, Virgisporangium, Actinomycetospora, Planotetraspora, and Cryptosporangium, were found only on Iriomote. Conversely, two genera, Oerskovia and Actinocorallia, were found only on Rishiri.

A total of 306 species were found among the Rishiri and Iriomote isolates. Of these, only $66(21.6 \%)$ were found on both Rishiri and Iriomote. Species compositions of representative genera, as well as the species diversity index of each genus, are shown in Table 4. The species diversities of Micromonospora and Streptomyces among the Iriomote isolates were significantly higher $(\mathrm{P}<0.05)$ than those of the Rishiri isolates. On the contrary, diversity of Kitasatospora species among Rishiri isolates was significantly higher $(\mathrm{P}<0.05)$ than that of the Iriomote isolates. Among the Rishiri Streptomyces isolates, strains of S. mauvecolor (13 strains), S. mirabilis (13 strains), and S. olivochromogenes (25 strains), all of which belong to the $S$. diastaticus phenotypic cluster of Williams et al. (1983), were found frequently. Several non-Streptomyces species, such as Actinoplanes philippinensis (Couch, 1950), Actinomadura 
Table 3. Actinomycete genera found in soils on Rishiri (a cool-temperate island) and Iriomote (a subtropical island).

\begin{tabular}{|c|c|c|c|c|c|c|}
\hline \multirow[b]{2}{*}{ Family $^{\text {b) }}$} & \multirow[b]{2}{*}{ Genus } & \multicolumn{2}{|c|}{ No. of isolates } & \multicolumn{3}{|c|}{ No. of species } \\
\hline & & Rishiri & Iriomote & Rishiri & Iriomote & $\begin{array}{l}\text { Both } \\
\text { islands }\end{array}$ \\
\hline \multirow[t]{3}{*}{ Actinosynnemataceae } & Lechevalieria & & 1 (1) & & & \\
\hline & Lentzea & 2 & $2(1)$ & 2 & 1 & \\
\hline & Unidentified & 1 (1) & & & & \\
\hline Brevibacteriaceae & Brevibacterium & & 1 & & 1 & \\
\hline Catenulisporaceae & Catenulispora & $1(1)^{\mathrm{c})}$ & $\left.1(1)^{\mathrm{c}}\right)$ & & & \\
\hline \multirow[t]{3}{*}{ Cellulomonadaceae } & Cellulomonas & 3 & 1 & 3 & 1 & \\
\hline & Oerskovia & 5 & & 2 & & \\
\hline & Unidentified & $2(2)$ & 1 (1) & & & \\
\hline Cryptosporangiaceae & Cryptosporangium & & 3 & & 2 & \\
\hline Glycomycetaceae & Glycomyces & & 1 & & 1 & \\
\hline \multirow[t]{6}{*}{ Intrasporangiaceae } & Humihabitans & 1 & 4 & 1 & 1 & $(1)^{\mathrm{d})}$ \\
\hline & Intrasporangium & & 1 & & 1 & \\
\hline & Oryzihumus & & 2 & & 1 & \\
\hline & Phycicoccus & & 1 (1) & & & \\
\hline & Terrabacter & 7 & 2 & 3 & 2 & (1) \\
\hline & Unidentified & 1 (1) & & & & \\
\hline Kineosporiaceae & Kineosporia & 5 & 1 (1) & 1 & & \\
\hline \multirow[t]{4}{*}{ Microbacteriaceae } & Agromyces & 2 & 19 (17) & 1 & 2 & \\
\hline & Labedella & & 1 (1) & & & \\
\hline & Microbacterium & 5 & 3 (2) & 4 & 1 & (1) \\
\hline & Unidentified & & 1 (1) & & & \\
\hline Micrococcaceae & Arthrobacter & 7 (2) & 1 (1) & 3 & & \\
\hline \multirow[t]{15}{*}{ Micromonosporaceae } & Actinoplanes & $44 \quad(20)$ & $42 \quad(27)$ & 8 & 7 & (3) \\
\hline & Asanoa & 4 (1) & 12 (2) & 2 & 2 & (2) \\
\hline & Catellatospora & 12 (5) & 9 & 5 & 3 & (3) \\
\hline & Catenuloplanes & & 7 & & 2 & \\
\hline & Couchioplanes & 2 & 11 & 1 & 2 & (1) \\
\hline & Dactylosporangium & 21 & 26 & 2 & 5 & (2) \\
\hline & Hamadaea & & 1 (1) & & & \\
\hline & Krasilnikovia & & $3(1)$ & & 1 & \\
\hline & Luedemannella & 1 (1) & $5 \quad(3)$ & & 1 & \\
\hline & Micromonospora & 22 & $63 \quad(5)$ & 12 & 19 & (10) \\
\hline & Pseudosporangium & 1 & 3 & 1 & 1 & (1) \\
\hline & Spirilliplanes & & $1 \quad(1)$ & & & \\
\hline & Verrucosispora & & $3(1)$ & & 1 & \\
\hline & Virgisporangium & & $5 \quad(4)$ & & 1 & \\
\hline & Unidentified & 1 (1) & $2(2)$ & & & \\
\hline Mycobacteriaceae & Mycobacterium & 2 & $7 \quad(3)$ & 2 & 4 & (1) \\
\hline
\end{tabular}


Continued.

\begin{tabular}{|c|c|c|c|c|c|c|}
\hline \multirow[b]{2}{*}{ Family ${ }^{\text {) }}$} & \multirow[b]{2}{*}{ Genus } & \multicolumn{2}{|c|}{ No. of isolates } & \multicolumn{3}{|c|}{ No. of species } \\
\hline & & Rishiri & Iriomote & Rishiri & Iriomote & $\begin{array}{l}\text { Both } \\
\text { islands }\end{array}$ \\
\hline \multirow[t]{4}{*}{ Nocardiaceae } & Gordonia & $7(6)$ & 2 & 1 & 2 & (1) \\
\hline & Nocardia & $52 \quad(17)$ & 31 & 13 & 12 & (2) \\
\hline & Rhodococcus & $25 \quad(10)$ & $20 \quad(8)$ & 6 & 7 & (3) \\
\hline & Unidentified & & 1 (1) & & & \\
\hline \multirow[t]{4}{*}{ Nocardioidaceae } & Kribbella & 12 & 4 & 6 & 3 & (3) \\
\hline & Marmoricola & & 1 (1) & & & \\
\hline & Nocardioides & 3 (2) & 4 (2) & 1 & 2 & \\
\hline & Unidentified & & 1 (1) & & & \\
\hline Nocardiopsaceae & Nocardiopsis & 8 (1) & 4 (1) & 3 & 3 & (1) \\
\hline \multirow[t]{4}{*}{ Promicromonosporaceae } & Cellulosimicrobium & 1 (1) & 1 & & 1 & \\
\hline & Isoptericola & 3 (1) & 16 & 1 & 3 & (1) \\
\hline & Myceligenerans & & 1 & & 1 & \\
\hline & Promicromonospora & & 1 & & 1 & \\
\hline \multirow[t]{5}{*}{ Pseudonocardiaceae } & Actinomycetospora & & 4 (4) & & & \\
\hline & Amycolatopsis & $6 \quad(2)$ & 1 (1) & 1 & & \\
\hline & Pseudonocardia & $5 \quad(4)$ & $17 \quad(5)$ & 1 & 5 & (1) \\
\hline & Saccharopolyspora & 2 & 1 & 1 & 1 & \\
\hline & Unidentified & 3 (3) & $2(2)$ & & & \\
\hline \multirow[t]{4}{*}{ Streptomycetaceae } & Kitasatospora & 27 & 3 & 7 & 1 & \\
\hline & Streptacidiphilus & 6 & $2(1)$ & 1 & 1 & \\
\hline & Streptomyces & $275 \quad(65)$ & $129(21)$ & 66 & 66 & $(23)$ \\
\hline & Unidentified & $3(3)$ & & & & \\
\hline \multirow[t]{7}{*}{ Streptosporangiaceae } & Acrocarpospora & & $2(2)$ & & & \\
\hline & Microbispora & $15 \quad(6)$ & 10 & 3 & 1 & (1) \\
\hline & Microtetraspora & 2 & 4 & 1 & 1 & \\
\hline & Nonomuraea & 7 (2) & 21 & 3 & 7 & (1) \\
\hline & Planotetraspora & & 3 (3) & & & \\
\hline & Sphaerisporangium & 1 & 8 & 1 & 1 & \\
\hline & Streptosporangium & $40 \quad(1)$ & 9 (1) & 4 & 4 & (2) \\
\hline \multirow[t]{2}{*}{ Thermomonosporaceae } & Actinocorallia & 1 & & 1 & & \\
\hline & Actinomadura & 12 & $15 \quad(2)$ & 4 & 7 & (1) \\
\hline \multirow[t]{2}{*}{ Tsukamurellaceae } & Tsukamurella & & 1 & & 1 & \\
\hline & & $668 \quad(161)$ & $566 \quad(150)$ & 178 & 194 & $(66)$ \\
\hline
\end{tabular}

\footnotetext{
${ }^{\text {a) }}$ Isolates were identified using a phylogenetic analysis based on comparison of nearly complete 16S rDNA sequences ( $>1,200$ bp).

b) Zhi et al. 2009.

c) Strains exhibiting <98.7\% 16S rDNA sequence similarity with a deposited sequence.

d) Number of species found both in Rishiri and Iriomote.
}

bangladeshensis (Ara et al., 2008), Dactylosporangium thailandense (Thiemann et al., 1967), Micromonospora chersina (Tomita et al., 1992), and M. narathiwatensis (Thawei et al., 2007), all of which have been isolated from tropical areas, were found only among the Iriomote isolates. Conversely, Actinoplanes cyaneus (Terekhova, et al., 1977), which had been reportedly isolated from a cold area (Siberia, Russia), was found among Rishiri isolates. 
Table 4. Species composition of representative genera found in soils on Rishiri (a cool-temperate island) and Iriomote (a subtropical island).

\begin{tabular}{|c|c|c|c|c|c|c|c|}
\hline \multirow[t]{2}{*}{ Family } & \multirow[t]{2}{*}{ Genus \& Species } & \multicolumn{2}{|c|}{$\begin{array}{l}\left.\text { No. of strains }{ }^{a}\right) \\
\text { (Diversity index) }\end{array}$} & \multirow[t]{2}{*}{ Family } & \multirow[t]{2}{*}{ Genus \& Species } & \multicolumn{2}{|c|}{$\begin{array}{l}\left.\text { No. of strains }{ }^{a}\right) \\
\text { (Diversity index) }\end{array}$} \\
\hline & & Rishiri & Iriomote & & & Rishiri & Iriomote \\
\hline \multirow{33}{*}{$\begin{array}{l}\text { Micromono- } \\
\text { sporaceae }\end{array}$} & Actinoplanes & & & Pseudonocardi- & Pseudonocardia & & \\
\hline & campanulatus & & 3 & aceae & carboxydivorans & & 3 \\
\hline & cyaneus & 8 & 1 & & halophobica & & 6 \\
\hline & digitatis & 4 & 6 & & Other species & 1 & 3 \\
\hline & durahamensis & 1 & & & (Shannon index) & $(0.00)$ & (1.90) \\
\hline & ferrugineus & 3 & & & & & \\
\hline & philippinensis & & 1 & Streptomycet- & Kitasatospora & & \\
\hline & regularis & 5 & & aceae & gansuensis & 4 & \\
\hline & Other species & 3 & 4 & & griseola & & 3 \\
\hline & (Shannon index) & $(2.57)$ & $(2.42)$ & & mediocidica & 13 & \\
\hline & & & & & nipponensis & 4 & \\
\hline & Dactylosporangium & & & & Other species & 6 & \\
\hline & aurantiacum & 17 & 8 & & (Shannon index) & $(2.23)^{*}$ & $(0.00)$ \\
\hline & fulvum & & 9 & & & & \\
\hline & matsuzakiense & 3 & 4 & & Streptomyces & & \\
\hline & roseum & & 3 & & atratus & 9 & 1 \\
\hline & thailandense & & 1 & & bungoensis & 5 & 4 \\
\hline & (Shannon index) & $(0.61)$ & $(2.03)$ & & carpaticus & & 3 \\
\hline & & & & & flavotricini & & 3 \\
\hline & Micromonospora & & & & glauciniger & 5 & 2 \\
\hline & carbonacea & 1 & 6 & & griseorubiginosus & 5 & \\
\hline & chaiyaphumensis & 1 & 12 & & hygroscopicus & 1 & 6 \\
\hline & chersina & & 4 & & lydicus & 8 & 7 \\
\hline & chokoriensis & 4 & 3 & & mauvecolor & 13 & \\
\hline & coxensis & 2 & 8 & & mirabilis & 13 & \\
\hline & echinospora & & 3 & & olivochromogenes & 25 & 1 \\
\hline & endolithica & & 2 & & paucisporeus & 4 & \\
\hline & matsumotoense & & 3 & & phaeolutei- & 1 & 3 \\
\hline & narathiwatensis & & 2 & & chromatogenes & & \\
\hline & siamensis & 4 & 1 & & prunicolor & 4 & \\
\hline & Other species & 9 & 24 & & psammoticus & & 4 \\
\hline & (Shannon index) & $(3.34)$ & $(3.81)^{* *}$ & & rishiriensis & 8 & \\
\hline & & & & & sanglieri & 12 & 2 \\
\hline \multirow[t]{14}{*}{ Nocardiaceae } & Nocardia & & & & spiroverticillatus & 4 & \\
\hline & araoensis & & 4 & & xanthochromo- & 4 & 2 \\
\hline & arthritidis & & 5 & & genes & & \\
\hline & brasiliensis & 3 & & & Other species & 89 & 70 \\
\hline & elegans & 3 & 6 & & (Shannon index) & $(5.38)$ & $(5.78)^{*}$ \\
\hline & harenae & 4 & & & & & \\
\hline & miyunensis & 5 & & Streptosporan- & Nonomuraea & & \\
\hline & niigatensis & 2 & 4 & giaceae & candida & 2 & 5 \\
\hline & nova & 9 & & & dietziae & & 2 \\
\hline & pseudovaccinii & & 2 & & kuesteri & & 2 \\
\hline & Other species & 9 & 9 & & Other species & 3 & 3 \\
\hline & (Shannon index) & $(3.31)$ & $(3.27)$ & & (Shannon index) & $(1.52)$ & $(2.50)$ \\
\hline & Rhodococcus & & & & Streptosporangium & & \\
\hline & aetherivorans & & 3 & & album & 4 & \\
\hline
\end{tabular}


Continued.

\begin{tabular}{|c|c|c|c|c|c|c|c|}
\hline \multirow[t]{2}{*}{ Family } & \multirow[t]{2}{*}{ Genus \& Species } & \multicolumn{2}{|c|}{$\begin{array}{l}\text { No. of strains }{ }^{\mathrm{a})} \\
\text { (Diversity index) }\end{array}$} & \multirow[t]{2}{*}{ Family } & \multirow[t]{2}{*}{ Genus \& Species } & \multicolumn{2}{|c|}{$\begin{array}{l}\text { No. of strains }{ }^{a)} \\
\text { (Diversity index) }\end{array}$} \\
\hline & & Rishiri & Iriomote & & & Rishiri & Iriomote \\
\hline \multirow{12}{*}{$\begin{array}{l}\text { Nocardioid- } \\
\text { aceae }\end{array}$} & erythropolis & 1 & 4 & & amethystogenes & 6 & 1 \\
\hline & jostii & 3 & & & claviforme & & 4 \\
\hline & maanshanensis & 6 & 1 & & vulgare & 27 & 2 \\
\hline & wratislaviensis & 3 & 1 & & Other species & 3 & 1 \\
\hline & tukisamuensis & 1 & & & (Shannon index) & $(1.34)$ & $(1.75)$ \\
\hline & Other species & 3 & 5 & & & & \\
\hline & (Shannon index) & $(2.24)$ & $(2.52)$ & $\begin{array}{l}\text { Thermomono- } \\
\text { sporaceae }\end{array}$ & $\begin{array}{l}\text { Actinomadura } \\
\quad \text { bangladeshensis }\end{array}$ & & 2 \\
\hline & Kribbella & & & & citrea & 3 & \\
\hline & sancticallisti & 3 & & & maheshkhaliensis & 7 & 1 \\
\hline & sandramycini & 4 & & & viridis & & 4 \\
\hline & Other species & 5 & 4 & & Other species & 2 & 6 \\
\hline & (Shannon index) & $(2.36)$ & $(1.50)$ & & (Shannon index) & $(1.55)$ & $(2.62)$ \\
\hline
\end{tabular}

a) Strains exhibiting $\geq 98.7 \% 16 \mathrm{~S}$ rDNA sequence similarity to a deposited sequence.

${ }^{*},{ }^{* *}$ Value is significantly higher than that of control $\left({ }^{*} \mathrm{P}<0.05,{ }^{* *} \mathrm{P}<0.01\right.$; Wilcoxon signed-rank test for paired samples).
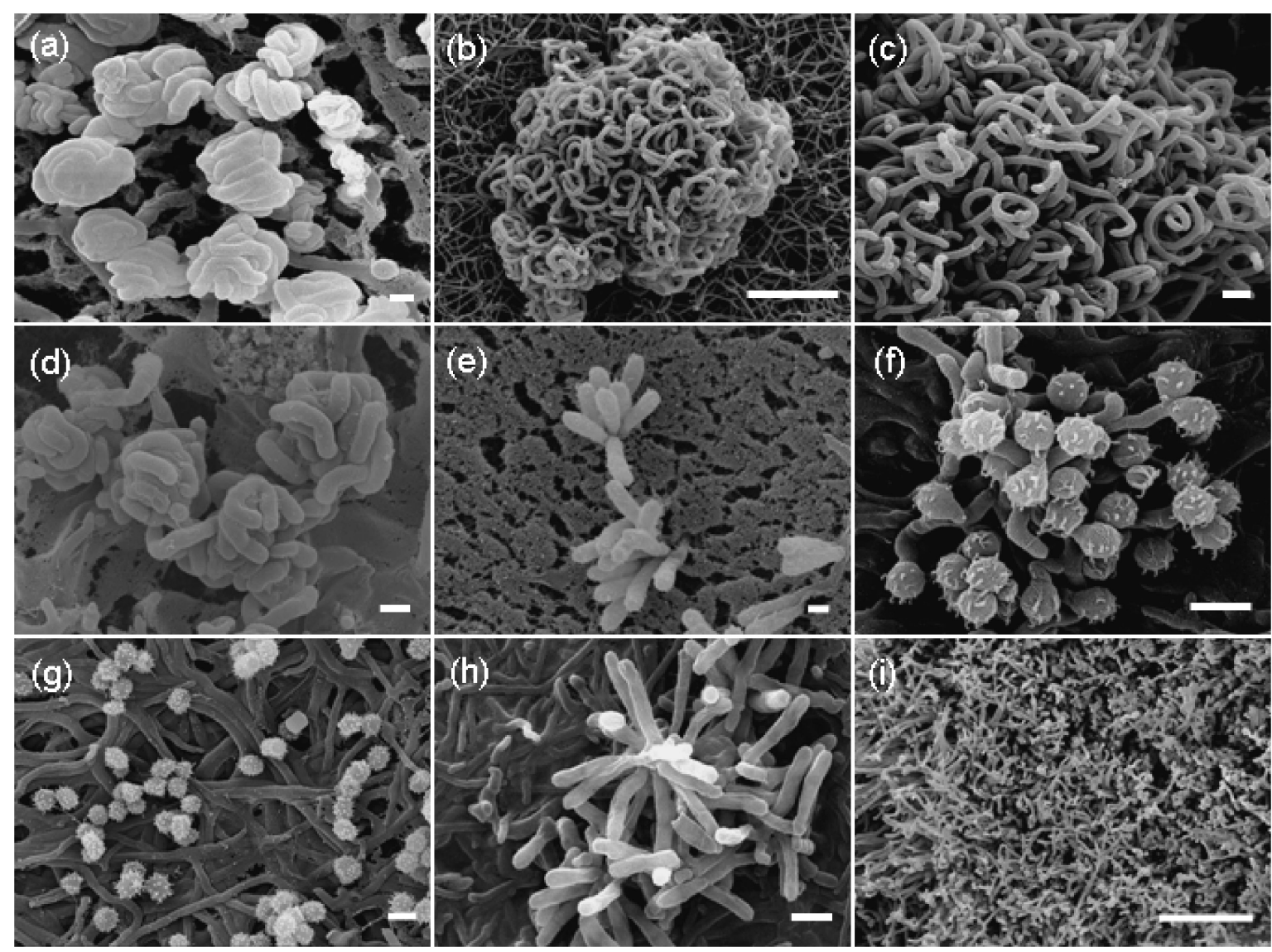

Fig. 2. Scanning electron micrographs of isolates belonging to the families Micromonosporaceae and Kineosporiaceae. (a) Actinoplanes sp. RI46-Co111; (b,c) Catenuloplanes sp. IR34-Co109; (d) Couchioplanes sp. IR27-RC105; (e) Dactylosporangium sp. IR33-SDHV105; (f) Micromonospora sp. IR19-RCA110; (g) Verrucosispora sp. IR10-DHHV603; (h) Virgisporangium sp. IR08-25; (i) Kineosporia sp. IR32-RC107. White bars: (a, c-g), $1 \mu \mathrm{m}$; (h), $2 \mu \mathrm{m}$; (i), $10 \mu \mathrm{m}$. 


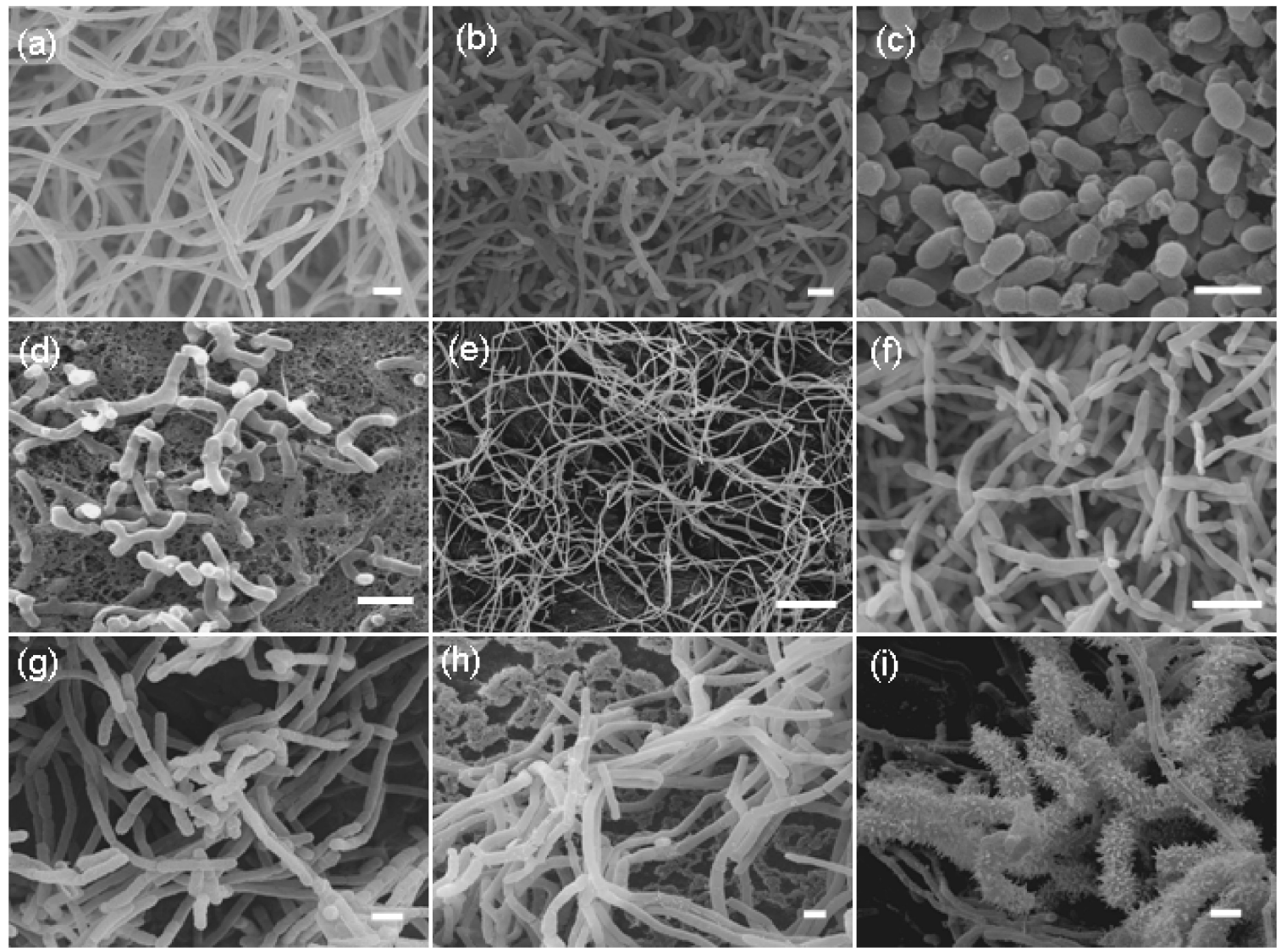

Fig. 3. Scanning electron micrographs of isolates belonging to the families Nocardioidaceae, Actinosynnemataceae, and Pseudonocardiaceae. (a) Kribbella sp. RI51-RC105; (b) Nocardioides sp. IR5-RCA101; (c) Marmoricola sp. IR-027S3; (d) Unidentified strain R19-10; (e) Lentzea sp. RI50-RCA110; (f) Actinomycetospora sp. IY07-53; (g) Amycolatopsis sp. RI50-SDHV701; (h) Pseudonocardia sp. IR11-SDHV701; (i) Saccharopolyspora sp. IR-27S2. White bars: (a-c, g-i), $1 \mu \mathrm{m}$; (d), $2 \mu \mathrm{m}$; (f), $5 \mu \mathrm{m}$; (e), $10 \mu \mathrm{m}$.

Scanning electron micrographs of representative actinomycete strains belonging to the families Micromonosporaceae, Kineosporiaceae, Nocardioidaceae, Actinosynnemataceae, Pseudonocardiaceae, Streptomycetaceae, Thermomonosporaceae, Nocardiopsaceae, Streptosporangiaceae, and Cryptosporangiaceae are shown in Fig. 2 to Fig. 4. Each micrograph shows fine morphology typical of the genus. For example, the micrograph of Catenuloplanes sp. IR34-Co109 (Fig. 2, b and c) shows the dichotomously branched aerial hyphae that are typical of the genus.

\section{Nucleotide sequence accession numbers}

The 16S rDNA sequences of selected isolates (42 strains representing 24 genera) that showed relatively low homology $(<98.5 \%)$ to prototype species or belonged to recently recognized rare genera are registered in the EMBL/ GenBank/DDBJ databases under accession numbers AB546272 to AB546313.

\section{DISCUSSION}

Although climate is believed to be an important ecological factor for microbial communities, very little is known about the effects of climate on the distribution of actinomycete species in soil. This lack of ecological information may be due to the difficulty in identifying actinomycetes at the species level. Polyphasic taxonomic approaches, including phenotypic characterization, chemotaxonomy, phylogenetic analysis based on 16S rDNA sequence, and a hybridization test of genomic DNA, are required for accurate species identification of actinomycetes. For ecological studies, however, systematic analysis of many actinomycete isolates is required. Therefore, a rapid and simple identification method is essential.

Simple molecular methods, such as the restriction fragment length polymorphism (RFLP) analysis of $16 \mathrm{~S}$ rDNA (Conville, et al., 2000; Yamamura, et al., 2003) and the repetitive extragenic palindromic (REP)-PCR fingerprinting technique (Olive \& Bean, 1999; Yamamura et al., 2004), have been suggested for characterization and classification of environmental actinomycetes. In recent years, with the progress in direct 16S rDNA sequencing techniques and the establishment of public-domain data- 

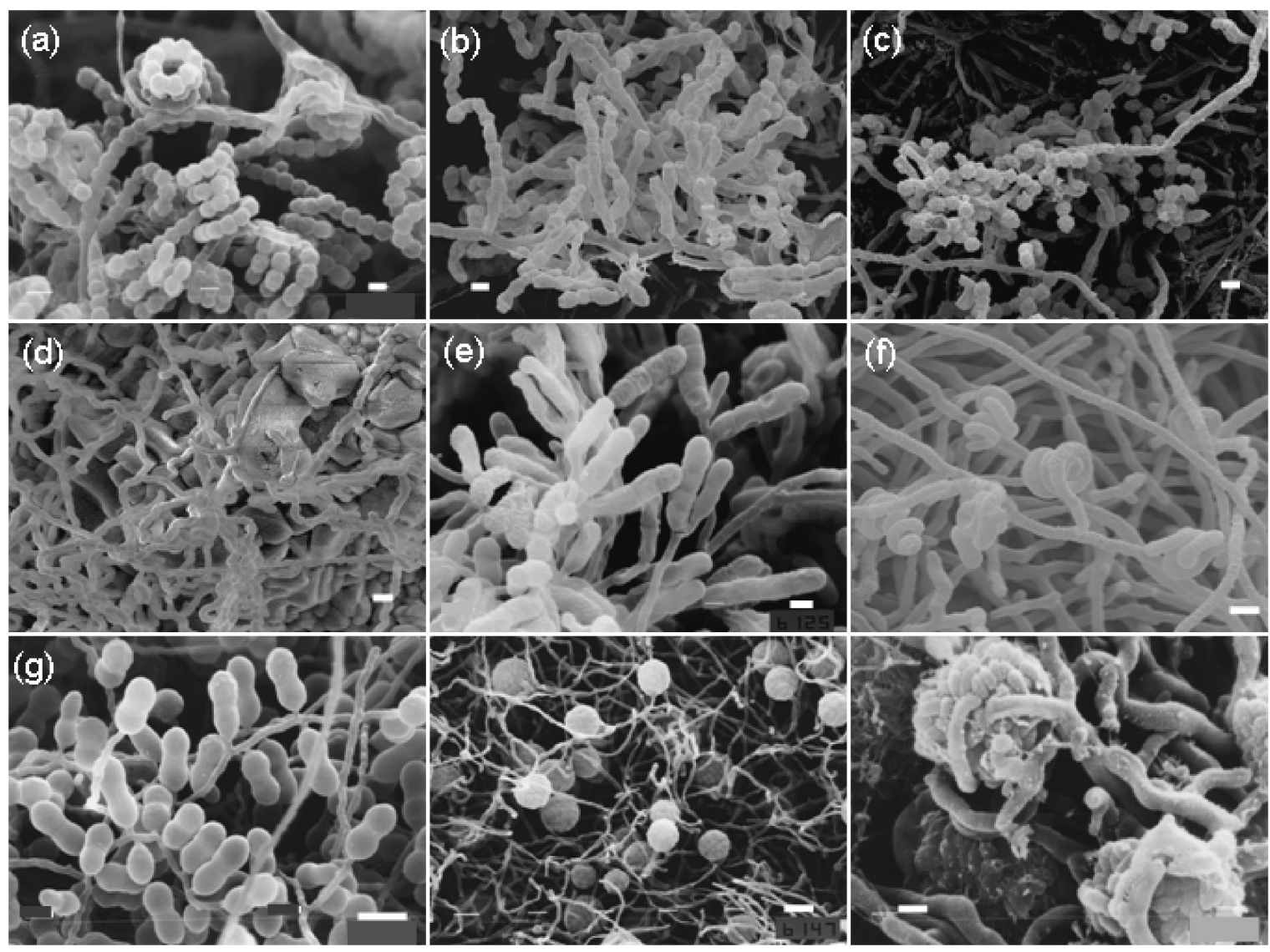

Fig. 4. Scanning electron micrographs of isolates belonging to the families Streptomycetaceae, Thermomonosporaceae, Nocardiopsaceae, Streptosporangiaceae, and Cryptosporangiaceae. (a) Streptomyces sp. IR01-03; (b) Actinocorallia sp. RI50-DHHV301; (c) Actinomadura sp. RI61-DHHV302; (d) Nocardiopsis sp. IR-21-N1; (e) Microtetraspora sp. TT07I-125; (f) Nonomuraea sp. RI69SDHV604; (g) Microbispora sp. IR12-DHHV201; (h) Streptosporangium sp. RI60-RC101; (i) Cryptosporangium sp. RI31-RCA103. White bars: (a-d, f, h), $1 \mu \mathrm{m}$; (e, g, i), $10 \mu \mathrm{m}$.

bases, 16S rDNA sequence comparison has been used successfully for simple identification at the genus or species level in ecological studies of actinomycetes (Wang et al., 1999; Muramatsu et al., 2003; Matsumoto, et al., 2006; Iwai, et al., 2009). In the present investigation, we demonstrated that $16 \mathrm{~S}$ rDNA-based identification is an efficient method of identification, with $98.5 \%$ of 1,234 actinomycete isolates being identified to the genus level and $74.8 \%$ being identified to the species level. These results are similar to those of Muramatsu et al., who identified $78.5 \%$ of 2,256 actinomycete isolates to the species level by a method based on partial $16 \mathrm{~S}$ rDNA sequence similarity. In our study, 19 isolates $(1.5 \%)$ could not be identified. These strains are considered prototype isolates of new genera or species, although further studies are needed for their accurate identification.

In this study, the Shannon diversity index results demonstrated that there was no significant difference in species diversity between Rishiri isolates and Iriomote isolates (Table 2). However, the fact that there was little taxonomic overlap between the Rishiri isolates and the
Iriomote isolates (Table 3) suggests that climate may influence the species types of soil-inhabiting actinomycetes. This hypothesis is supported by the fact that several species of tropical origin were isolated only from soils on the subtropical island, Iriomote.

The total number of actinomycete genera recovered (61 genera) is much higher than that in similar studies performed in other geographic areas (Wang et al., 1999; Muramatsu et al., 2003). The reason for this may be the use of different pre-treatment methods. Of these methods, the SDS method was particularly successful for isolation of actinomycetes including Streptomyces spp. on isolation plates while restricting the growth of impeding bacterial associates. Use of the dry-heating method, the rehydration and centrifugation method, and the chemotactic method enabled selective isolation of non-streptomycete actinomycetes. In previous studies, these organisms were recovered only incidentally using conventional dilution approaches. Progress in molecular methods has enabled the detection and characterization of actinomycetes in soil without their cultivation. However, the isolation of pure 
cultures of taxonomically diverse actinomycetes is still important to understand their relative abundance in the natural environment and to find novel strains that produce useful compounds (Goodfellow \& Williams, 1986). The actinomycete strains obtained in the present study could be important sources of novel bioactive compounds.

\section{ACKNOWLEDGMENTS}

We thank Professor Dr. T. Misonou and Associate Professor Dr. T. Shimokawa of University of Yamanashi for their helpful suggestions regarding statistical analysis. This study was supported in part by a research grant from the Institute for Fermentation, Osaka (IFO).

\section{REFERENCES}

Ara, I., Matsumoto, A., Bakir, M.A., Kudo, T., Ōmura, S. \& Takahashi, Y. (2008). Actinomadura bangladeshensis sp. nov. and Actinomadura chokoriensis sp. nov. Int. J. Syst. Evol. Microbiol. 58, 1653-1659.

Brosius, J., Palmer, J.L., Kennedy, J.P. \& Noller, H.F. (1978). Complete nucleotide sequence of a $16 \mathrm{~S}$ ribosomal RNA gene from Escherichia coli. Proc. Natl. Acad. Sci. U.S.A. 75, 48014805.

Chun, J., Lee, J.H, Jung, Y., Kim, M., Kim, S., Kim, B.K. \& Lim, Y.W. (2007). EzTaxon: a web-based tool for the identification of prokaryotes on 16S ribosomal RNA gene sequences. Int. J. Syst. Evol. Microbiol. 57, 2259-2261.

Conville, P.S., Fischer, S.H., Cartwright, C.P. \& Witebsky, F.G. (2000). Identification of Nocardia species by restriction endonuclease analysis of an amplified portion of the 16S rRNA gene. J. Clin. Microbiol. 38, 158-164.

Couch, J.N. (1950). Actinoplanes a new genus of the Actinomycetales. J. Elisha Mitchell Sci. Soc. 66, 8792.

Drancourt, M., Bollet, C., Carlioz, A., Martelin, R., Gayral, J.P. \& Raoult, D. (2000). $16 \mathrm{~S}$ ribosomal DNA sequence analysis of a large collection of environmental and clinical unidentifiable bacterial isolates. J. Clin. Microbiol. 38, 36233630.

Felsenstein, J. (1985). Confidence limits on phylogenies: an approach using the bootstrap. Evolution 39, 783-791.

Flanagan, J.L., Brodie, E.L., Weng, L., Lynch, S.V., Garcia, O., Brown, R., Hugenholz, P., DeSantis, T.Z., Andersen, G.L., Wiener-Kronish, J.P. \& Bristow, J. (2007). Loss of bacterial diversity during antibiotic treatment of intubated patients colonized with Pseudomonas aeruginosa. J. Clin. Microbiol. 45, 1954-1962.

Goodfellow, M. \& Williams, S.T. (1983). Ecology of actinomycetes. Ann. Rev. Microbiol. 37, 189-216.

Goodfellow, M. \& Williams, E. (1986). New strategies for the selective isolation of industrially important bacteria. Biotechnol. Genet. Eng. Rev. 4, 213-262.

Hayakawa, M. \& Nonomura, H. (1987). Humic acid-vitamin agar, a new medium for the selective isolation of soil actinomycetes. J. Ferment. Technol. 65, 501-509.

Hayakawa, M. \& Nonomura, H. (1989). A new method for the intensive isolation of actinomycetes from soil. Actinomycetologica 3, 95-104.
Hayakawa, M., Iino, S. \& Nonomura, H. (1982). Heavy metal resistance and melanoid pigment production in the streptomycete flora of copper-polluted vineyard soils. J. Ferment. Technol. 60, 1-10.

Hayakawa, M., Ishizawa, K. \& Nonomura, H. (1988). Distribution of rare actinomycetes in Japanese soils. J. Ferment. Technol. 66, 367-373.

Hayakawa, M., Sadakata, T., Kajiura, T. \& Nonomura, H. (1991a). New methods for the highly selective isolation of Micromonospora and Microbispora from soil. J. Ferment. Bioeng. 72, 320-326.

Hayakawa, M., Tamura, T. \& Nonomura, H. (1991b). Selective isolation of Actinoplanes and Dactylosporangium from soil by using $\gamma$-collidine as the chemoattractant. J. Ferment. Bioeng. 72, 426-432.

Hayakawa, M., Momose, Y., Yamazaki, T. \& Nonomura, H. (1996). A method for the selective isolation of Microtetraspora glauca and related four-spored actinomycetes from soil. $J$. Appl. Bacteriol. 80, 375-386.

Hayakawa, M., Otoguro, M., Takeuchi, T., Yamazaki, T. \& Iimura, Y. (2000). Application of a method incorporating differential centrifugation for selective isolation of motile actinomycetes in soil and plant litter. Ant. Leeuwenhoek 78, 171-185.

Iwai, K., Iwamoto, S., Aisaka, K. \& Suzuki, M. (2009). Isolation of novel actinomycetes from spider materials. Actinomycetologica 23, 8-15.

Jones, K.L. (1949). Fresh isolates of actinomycetes in which the presence of sporogenous aerial mycelium is a fluctuating characteristic. J. Bacteriol. 57, 141-145.

Matsumoto, A., Takahashi, Y., Iwai, Y. \& Ōmura, S. (2006). Isolation of Gram-positive bacteria with high $\mathrm{G}+\mathrm{C}$ from inside soil aggregates. Actinomycetologica 20, 30-34.

Muramatsu, H., Shahab, N., Tsurumi, Y. \& Hino, M. (2003). A comparative study of Malaysian and Japanese actinomycetes using a simple identification method based on partial 16S rDNA sequence. Actinomycetologica 17, 33-43.

Nonomura, H. \& Ohara, Y. (1969a). Distribution of actinomycetes in soil. VII. A culture method effective for both preferential isolation and enumeration of Microbispora and Streptosporangium strains in soil. (Part 1) Classification of the isolates. J. Ferment. Technol. 47, 463-469.

Nonomura, H. \& Ohara, Y. (1969b). Distribution of actinomycetes in soil. VII. A culture method effective for both preferential isolation and enumeration of Microbispora and Streptosporangium strains in soil. (Part 2) Classification of the isolates. J. Ferment. Technol. 47, 701-709.

Nonomura, H., Iino, S. \& Hayakawa, M. (1979). Classification of actinomycete genus Ampullariella from soils of Japan. $J$. Ferment. Technol. 57, 79-85.

Olive, D.M. \& Bean, P. (1999). Principles and applications of methods for DNA-based typing of microbial organisms. J. Clin. Microbiol. 37, 1661-1669.

Saitou, N. \& Nei, M. (1987). The neighbor-joining method: a new method for reconstructing phylogenetic trees. Mol. Biol. Evol. 4, 406-425.

Shannon, C.E. \& Weaver, W. (1949). The mathematical theory of communication. University of Illinois Press, Urbana, Ill.

Stackebrandt, E. \& Ebers, J. (2006). Taxonomic parameters revisited: tarnished gold standards. Microbiol. Today Nov 06, 152-155. 
Tamura, T. \& Hatano, K. (2001). Phylogenetic analysis of the genus Actinoplanes and transfer of Actinoplanes minutisporangius Ruan et al. 1986 and 'Actinoplanes aurantiacus' to Cryptosporangium minutisporangium comb. nov. and Cryptosporangium aurantiacum sp. nov. Int. J. Syst. Evol. Microbiol. 51, 2119-2125.

Terekhova, L.P., Sadikova, O.A. \& Preobrazhenskaia, T.P. (1977). New species of Actinoplanes cyaneus sp. nov. and its antagonistic properties. Antibiotiki 22, 1059-1063.

Thawai, C., Tanasupawat, S., Suwanborirux, K, Itoh, T. \& Kudo, T. (2007). Micromonospora narathiwatensis sp. nov., from Thai peat swamp forest soils. J. Gen. Appl. Microbiol. 53, 287-293.

Thiemann, J.E., Pagani, H. \& Beretta, G. (1967). A new genus of the Actinoplanaceae: Dactylosporangium gen. nov. Arch. Mikrobiol. 58, 42-52.

Thompson, J.D., Gibson, T.J., Plewniak, F., Jeanmougin, F. \& Higgins, D.G. (1997). The CLUSTAL_X windows interface: flexible strategies for multiple sequence alignment aided by quality analysis tools. Nucleic Acids Res. 25, 4876-4882.

Tomita, K., Hoshino, Y., Ohkusa, N., Tsuno, T. \& Miyaki, T. (1992). Micromonospora chersina sp. nov. Actinomycetologica 6, 21-28.

Wang, Y., Zhang, Z.S., Ruan, J.S., Wang, Y.M. \& Ali, S.M. (1999). Investigation of actinomycete diversity in the tropical rainforests of Singapore. J. Ind. Microbiol. Biotechnol. 23, 178-
187.

Williams, S.T., Goodfellow, M., Alderson, G., Wellington, E.M.H., Sneath, P.H.A. \& Sackin, M.J. (1983). Numerical classification of Streptomyces and related genera. J. Gen. Microbiol. 129, 1743-1813.

Williams, S.T., Lanning, S. \& Wellington, E.M.H. (1984). Ecology of actinomycetes. In The Biology of Actinomycetes (eds. Goodfellow, M., Mordarski, M. and Williams, S.T.) pp. 481-528, Academic Press, London.

Xu, L.H., Li, Q.R. \& Jiang, C.L. (1996). Diversity of soil actinomycetes in Yunnan, China. Appl. Environ. Microbiol. 62, 244-248.

Yamamura, H., Hayakawa, M., Nakagawa, Y. \& Iimura, Y. (2003b). Species diversity of Nocardiae isolated from lake and moat sediment samples. Actinomycetologica 17, 44-46.

Yamamura, H., Hayakawa, M., Nakagawa, Y. \& Iimura, Y. (2004). Characterization of Nocardia asteroides isolates from different ecological habitats based on repetitive extragenic palindromic-PCR fingerprinting. Appl. Environ. Microbiol. 70, 3149-3151.

Zhi, X.Y., Li, W.J. \& Stackebrandt, E. (2009). An update of the structure and 16S rRNA gene sequence-based definition of higher ranks of the class Actinobacteria, with the proposal of two new suborders and four new families and emended descriptions of the existing higher taxa. Int. J. Syst. Evol. Microbiol. 59, 589-608. 